\title{
Does preoperative chemo-radiotherapy enhance the expression of vascular endothelial growth factor in patients with rectal cancer?
}

\author{
YASUHIRO INOUE, EIKI OJIMA, HIDEKI WATANABE, JUNICHIRO HIRO, YUJI TOIYAMA, \\ MINAKO KOBAYASHI, CHIKAO MIKI and MASATO KUSUNOKI \\ Division of Reparative Medicine, Department of Gastrointestinal and Pediatric Surgery, Institute of \\ Life Sciences, Mie University Graduate School of Medicine, Mie 514-8507, Japan
}

Received March 12, 2007; Accepted May 25, 2007

\begin{abstract}
To assess whether preoperative chemo-radiotherapy enhances the expression of vascular endothelial growth factor (VEGF) in patients with colorectal cancer, we investigated in vivo and in vitro the interactions between chemoradiotherapy and the expression of VEGF, and their possible impact on distant metastasis. Cellular cytotoxicity in the colon cancer cell lines, LoVo, SW480 and Caco2, was determined using a WST-8 colorimetric assay after cells were exposed to 5-fluorouracil combined with radiation. In addition, the VEGF levels in cultured cells were measured by ELISA. Preoperative serum samples and tumor specimens were prospectively collected from 32 rectal cancer patients who received preoperative chemo-radiotherapy. Both local and circulating VEGF expression levels were measured perioperatively by ELISA, and assessed in relation to the clinicopathological findings. Perioperative circulating VEGF levels from irradiated patients were also compared with a nonirradiated control group. There were significant increases in local VEGF levels, both in vivo and in vitro, after chemoradiotherapy, especially in viable cancer cells. The circulating VEGF levels in the irradiated patients were significantly lower after surgery compared with those in the control group. Although preoperative chemo-radiotherapy enhanced tumorspecific VEGF expression, especially in individual cancer cells both in vitro and in vivo, it did not necessarily enhance systemic VEGF expression, possibly because of tumor volume reduction induced by the chemo-radiotherapy.
\end{abstract}

Correspondence to: Dr Masato Kusunoki, Division of Reparative Medicine, Department of Gastrointestinal and Pediatric Surgery, Institute of Life Sciences, Mie University Graduate School of Medicine, Edobashi 2-174, Tsu, Mie 514-8507, Japan

E-mail: kusunoki@clin.medic.mie-u.ac.jp

Key words: rectal cancer, preoperative chemo-radiotherapy, vascular endothelial growth factor

\section{Introduction}

Over the past three decades, (neo-)adjuvant radiotherapy with or without chemotherapy has been widely used in attempts to improve outcomes in rectal cancer. The National Cancer Institute Consensus Conference in the United States in 1990 recommended post-operative chemo-radiotherapy for patients with TNM stage II and III rectal cancers as standard treatment (1). A particular dose of irradiation appears to be more effective if given preoperatively rather than post-operatively, probably because oxygen tension within the tumor may be higher prior to the surgical compromise of regional blood flow (2).

Several randomized trials reported a benefit of preoperative radiotherapy compared to post-operative treatment (3-6). The results of these trials demonstrated clearly the superiority of preoperative radiotherapy in terms of local control, with better compliance to treatment and lower toxicity. A recent metaanalysis also concluded that the combination of preoperative radiotherapy and surgery, as compared with surgery alone, significantly improves local control and overall survival $(7,8)$.

However, the exact role of preoperative radiotherapy remains controversial for several reasons, especially in terms of its benefit to survival, as distant metastasis remains a significant problem even after preoperative radiation therapy $(9,10)$. Recently, chemotherapy has been added to radiotherapy in an attempt to resolve this problem. Indeed, some studies have shown the effectiveness of preoperative chemoradiotherapy for resectable rectal cancer, not only by reducing rates of local recurrence, but also by improving survival $(11,12)$. Although chemo-radiotherapy for resectable rectal cancer has now been widely accepted, current practices such as radiation technique (conventional fractionation or short course radiotherapy) and chemotherapy regimen differ between countries, and even between institutions within the same country. Thus, the issue of adjuvant treatment for rectal cancer is one of the major controversies in the field of oncology.

Various cytokines or growth factors play important roles in carcinogenesis and tumor proliferation. In particular, vascular endothelial growth factor (VEGF) is a multifunctional cytokine that potently stimulates angiogenesis, including tumor neovascularization (13). Recent studies have revealed 
Table I. Experimental schedule.

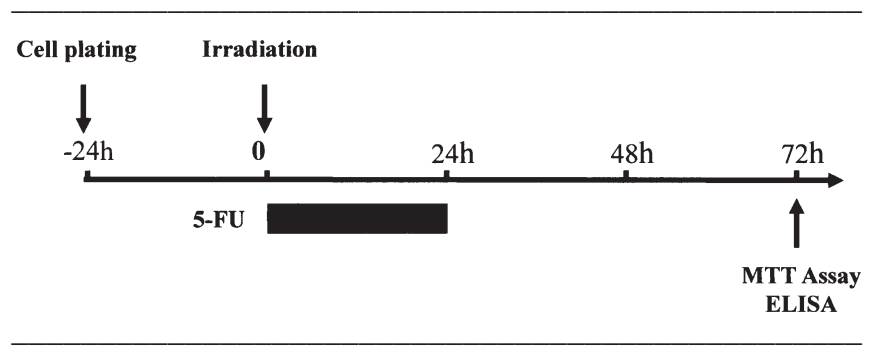

relationships among an increased expression of VEGF, distant metastasis, and poor prognoses of patients with colorectal cancer $(14,15)$. Induction of VEGF expression, in vivo and in vitro, after exposure to ionizing radiation, was recently reported $(16,17)$. Nozue et al also reported that over-expression of VEGF after preoperative radiotherapy was involved in distant metastasis (18).

This study evaluated the relationship between preoperative chemo-radiotherapy and the expression of VEGF in patients with rectal cancer, and its possible impacts on distant metastasis. We address the question: does preoperative chemo-radiotherapy enhance the tumor-specific and systemic expression of angiogenic growth factors in patients with rectal cancer?

\section{Materials and methods}

Three colon cancer cell lines, the p53 wild-type LoVo cells, and the mutant type SW480 and Caco 2 cells were used. The cell lines were grown in RPMI-1640 medium. The medium was supplemented with $10 \%$ fetal bovine serum, 100 units $/ \mathrm{ml}$ of penicillin and $100 \mu \mathrm{g} / \mathrm{ml}$ of streptomycin at $37^{\circ} \mathrm{C}, 5 \% \mathrm{CO}_{2}$. Cells were plated at a density of $1.0 \times 10^{4}$ cells $/ \mathrm{cm}^{2}$ plate area and grown for 4 days prior to further experimentation. The experiments were performed with exponentially growing cells.

Anticancer agent. 5-Fluorouracil (5-FU) was obtained from Sigma Aldrich (St. Louis, MO, USA). The drug was dissolved at appropriate concentrations in distilled water and stored at $-20^{\circ} \mathrm{C}$ until experiments.

Chemo-radiation procedure in vitro. Colon cancer cells were irradiated at room temperature. Cells were treated with 5 Gy of X-ray irradiation (100 kV and $3.5 \mathrm{~mA}$ for $5 \mathrm{~min})$ using an X-ray generator (MBR-1505R; Hitachi Medical Co., Tokyo, Japan). The chemo-radiation schedules are shown in Table I. The concentration of concurrent 5-FU was $10 \mu \mathrm{M}$ for $24 \mathrm{~h}$ with irradiation, according to our previous report (19). The time-point of 5-FU exposure was $0 \mathrm{~h}$ before irradiation.

Cytotoxicity assay. 3-(4,5-Dimethylthiazol-2-yl)-2,5-dipheniltetrazoliumbromide (MTT) assays were used to determine the response of tumor cells to irradiation. Cells were seeded in 96-well plates at a density of $1.0 \times 10^{3}$ cells $/ \mathrm{cm}^{2}$, treated with or without 5 Gy of X-ray irradiation, and incubated. Viable cells were measured by MTT assays at $72 \mathrm{~h}$ after irradiation.
Patient cohort. A total of 32 patients (22 males and 10 females aged 48-77 years; mean, 62.2) with rectal cancer, admitted to Mie University Hospital between 2001 and 2006, were enrolled in the study. The clinical backgrounds of patients are shown in Table II. All patients received preoperative chemo-radiotherapy. Treatment was by external irradiation (10 MV photons from a linear accelerator) using a four-field box technique and patients received 20 Gy in 4 fractions over a period of 1 week. The irradiation field included the entire sacrum, pubic bones, and the medial portion of the ilium. Patients also underwent concurrent pharmacokinetic modulating chemotherapy (PMC; intravenous infusion of 5-FU, $750 \mathrm{mg} / \mathrm{day}$, and oral administration of UFT, $400 \mathrm{mg} /$ day) over a period of 1 week (20). The rectal tumors were biopsied by endoscopy for histopathological diagnosis before irradiation. All 32 tumors were diagnosed as adenocarcinoma and the patients underwent chemo-radiotherapy. All patients underwent curative resection, an average of 10 days after chemo-radiotherapy was completed. The perioperative circulating VEGF levels from the irradiated patients were also compared with those from 30 matched rectal cancer patients who underwent curative surgery without neoadjuvant chemo-radiotherapy (Table II).

ELISA of VEGF expression. A supernatant of cultured cells was collected $72 \mathrm{~h}$ after irradiation and stored at $-80^{\circ} \mathrm{C}$ until use. Surgical specimens were immediately stored in liquid nitrogen until use. Tissues were homogenized and collected.

Peripheral venous blood samples were also collected into sterile glass tubes, permitted to coagulate at room temperature, and then centrifuged at $3000 \mathrm{x} \mathrm{g}$ for $5 \mathrm{~min}$. Serum was separated, aliquoted, and stored at $-80^{\circ} \mathrm{C}$ until use. Serum samples from patients were collected pre- and post-irradiation, and at 1, 3 and 7 days after operation. Serum samples were also collected perioperatively from rectal cancer patients who underwent curative surgery without chemo-radiotherapy.

The supernatants of cultured cells, tissue and serum were used to measure the VEGF concentrations using a commercially available enzyme linked immunosorbent assay (ELISA) kit (Human VEGF Immunoassay; BioSource International, Inc.). The protein concentration was measured using a BCA Protein Assay Regent Kit (Pierce Chemical Co., Rockford, IL).

Immunohistochemical staining of VEGF. Specimens in paraffin blocks were cut into $5-\mu \mathrm{m}$ sections and attached to glass slides with wax melted at $65^{\circ} \mathrm{C}$. The sections were then dewaxed, hydrated and incubated in $3 \%$ hydrogen peroxide for $30 \mathrm{~min}$. Next, they were washed in cold tap water, microwaved, and washed three times in $\mathrm{pH} 7.4$ phosphate-buffered saline (PBS) for $5 \mathrm{~min}$. After washing with PBS, sections were incubated with primary antibodies at room temperature for $2 \mathrm{~h}$. Non-specific binding was blocked by incubation with $3 \%$ normal goat serum for $20 \mathrm{~min}$. Sections were then incubated with a rabbit polyclonal antibody raised against VEGF (Santa Cruz Biotechnology, Santa Cruz, CA) at a dilution of $1: 100$, for $2 \mathrm{~h}$ at room temperature. The sections were washed again and incubated for $30 \mathrm{~min}$ at room temperature with biotinylated anti-rabbit IgG diluted in PBS. They were then incubated with Vector DAB substrate for $1.5 \mathrm{~min}$ and counterstained with Meyer's hematoxylin. The specificity of the immunoreactivity was verified by staining known 
Table II. Clinical backgrounds of rectal cancer patients.

\begin{tabular}{|c|c|c|c|}
\hline & Irradiated patients & $\begin{array}{l}\text { Non-irradiated } \\
\text { patients (control) }\end{array}$ & P-value \\
\hline No. of patients & 32 & 30 & \\
\hline \multicolumn{4}{|l|}{ Gender } \\
\hline Male & 22 & 22 & ns \\
\hline Female & 10 & 8 & \\
\hline Age (mean) & 62.2 & 62.8 & ns \\
\hline \multicolumn{4}{|l|}{ TNM classification } \\
\hline I & 7 & 7 & ns \\
\hline II & 5 & 7 & \\
\hline III & 20 & 16 & \\
\hline \multicolumn{4}{|l|}{$\begin{array}{l}\text { Histologic differentiation } \\
\text { (before chemo-radiation) }\end{array}$} \\
\hline Well & 5 & 14 & ns \\
\hline Moderately & 23 & 15 & \\
\hline Poorly & 2 & 1 & \\
\hline Mucinous & 2 & 0 & \\
\hline \multicolumn{4}{|l|}{ Type of surgery } \\
\hline Low anterior resection & 12 & 16 & $\mathrm{~ns}$ \\
\hline Hartmann's procedure & 1 & 2 & \\
\hline Abdominoperitoneal rectal resection & 2 & 10 & \\
\hline Anoabdominal rectal resection & 15 & 2 & \\
\hline Radiation effect (mean) & 0.39 & - & \\
\hline \multicolumn{4}{|l|}{ Histological grading } \\
\hline Grade 0 & 1 & - & \\
\hline Grade 1a & 10 & - & \\
\hline Grade 1b & 10 & - & \\
\hline Grade 2 & 8 & - & \\
\hline Grade 3 & 3 & - & \\
\hline
\end{tabular}

ns, not statistically significant.

positive and negative control tissue sections, and also by negative staining when the primary antibody was replaced with normal rabbit serum.

Radiation effects. The effects of radiation on tumor cells were judged from the number or amount of viable cells remaining in the specimen. A quantitative morphologic measure of radiation effects was performed on each specimen to determine the proportion of tumor cells to the background stroma, using an image analyzing system (Win Roof Version 3.6; Mitani Corp., Japan) as previously reported (21). Data for each tumor represent the mean value from three sections. Radiation effect was calculated from the ratio of residual tumor nests/ (residual tumor nests + background stroma) to correct for the VEGF produced by viable cancer cells.
The histological grading employed in this series was in accord with the General Rules of the Japanese Research Society for Cancer of the Colon and Rectum (JRSCCR) (22) as follows: Grade 0, tumor structures have not been destroyed; Grade $1 \mathrm{a},<1 / 3$ of the whole lesion has been destroyed; Grade $1 b,<2 / 3$ of the whole lesion has been destroyed; Grade 2, $>2 / 3$ of the whole lesion has been destroyed; Grade 3, no tumor cells remain in any of the sections.

Statistical methods. The relationships between VEGF expression and various clinico-pathological factors were tested using a $\chi^{2}$ test and a Mann-Whitney $U$ test. Statistical significance was defined as $p$-values of $<0.05$. Stat View analysis software (version 5; Abacus Concepts, Inc., Berkeley, CA) was used for all analysis. 

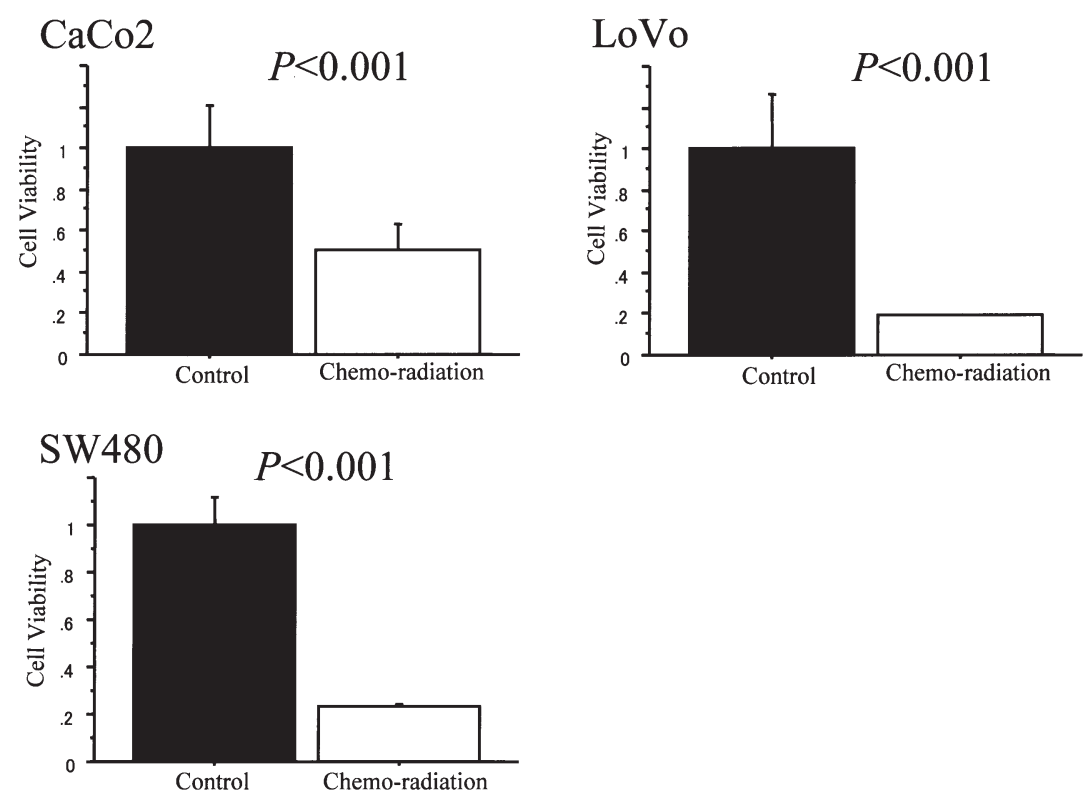

Figure 1. Cell viability was significantly decreased after chemo-radiation compared to control cells.

\section{$\mathrm{Caco} 2$}

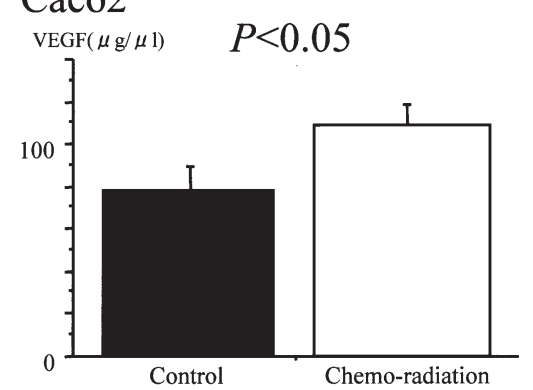

$\underset{\operatorname{VEGF}(\mu \mathrm{g} / \mu \mathrm{ll})}{\mathrm{SW} 480}$

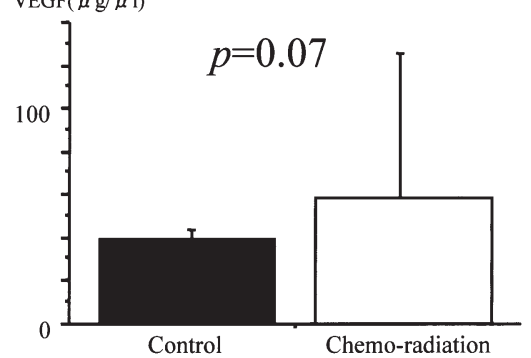

LoVo

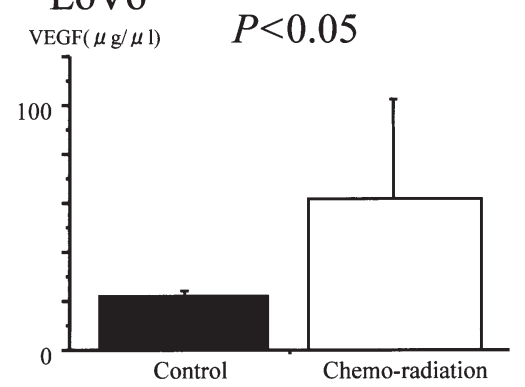

Figure 2. The VEGF levels in cultured cells are significantly increased in LoVo and Caco2 cells after chemo-radiation, when the VEGF levels are corrected for the number of viable cancer cells.

\section{Results}

Cell viability of colon cancer cells by chemo-radiation. The cytotoxic effects of chemo-radiation in LoVo, SW480 and Caco 2 cells were assessed at $72 \mathrm{~h}$ after chemo-radiation by the MTT assay. In all cell lines, cell viability was significantly lower after chemo-radiation than in control cells (Fig. 1).

VEGF levels in cultured cells. The VEGF levels in cultured cells were measured by ELISA and corrected for the number of residual cancer cells (the VEGF concentration of super- natant/cell viability). The level of VEGF per residual cancer cell was increased in all cell lines after chemo-radiation, especially in LoVo and Caco2 cells (Fig. 2).

Chemo-radiation effects in human tissue. Chemo-radiation effects were calculated from the the ratio of residual tumor nests/(residual tumor nests + background stroma) using a WinRoof imaging analyzing system. In one case, chemoradiation was totally ineffective (Grade 0 ), while three cases showed a complete response (Grade 3 ). The distribution of the grades of radiation effect is shown in Table II. 

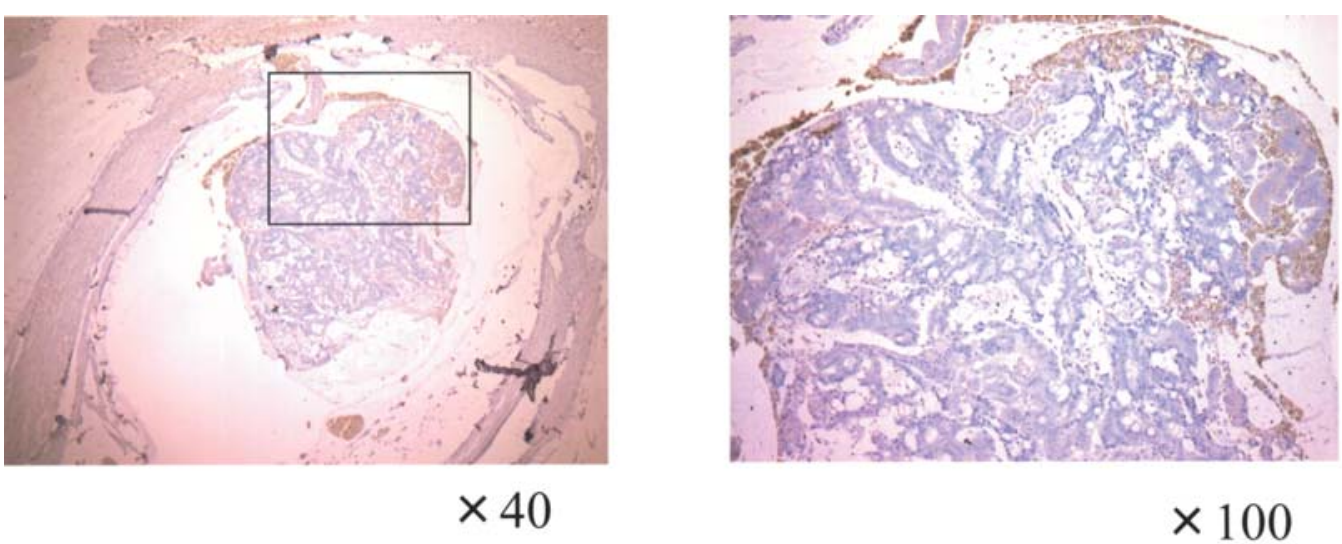

Figure 3. In a resected specimen, immunohistochemical staining reveals that VEGF expression is mainly localized in the cytoplasm or membrane of cancer cells.

a)

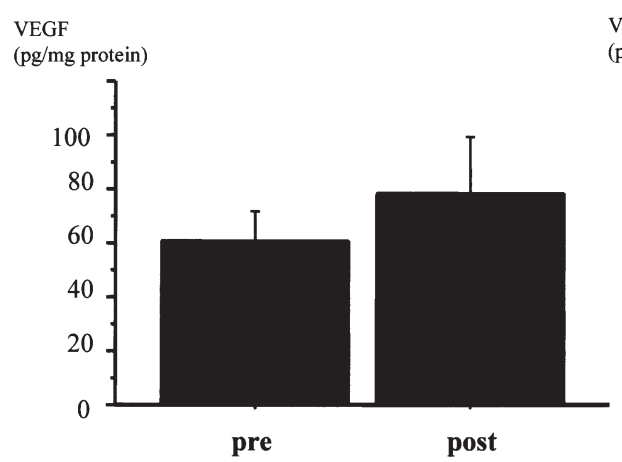

b)

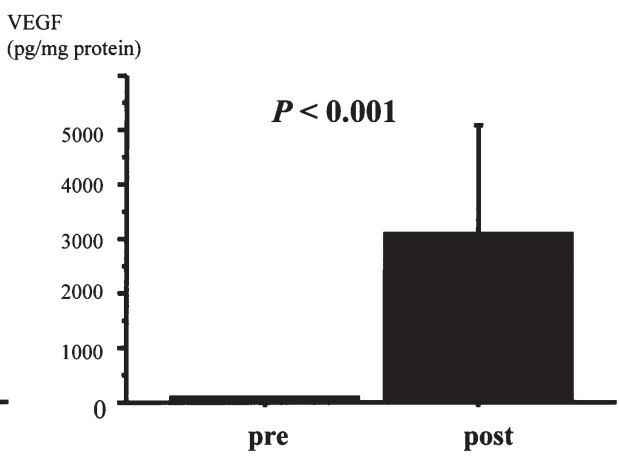

Figure 4. (a) The VEGF levels in human tissue are not significantly enhanced after chemo-radiation. (b) The VEGF levels corrected for the number of viable cells using the radiation effect are significantly increased after chemo-radiation.

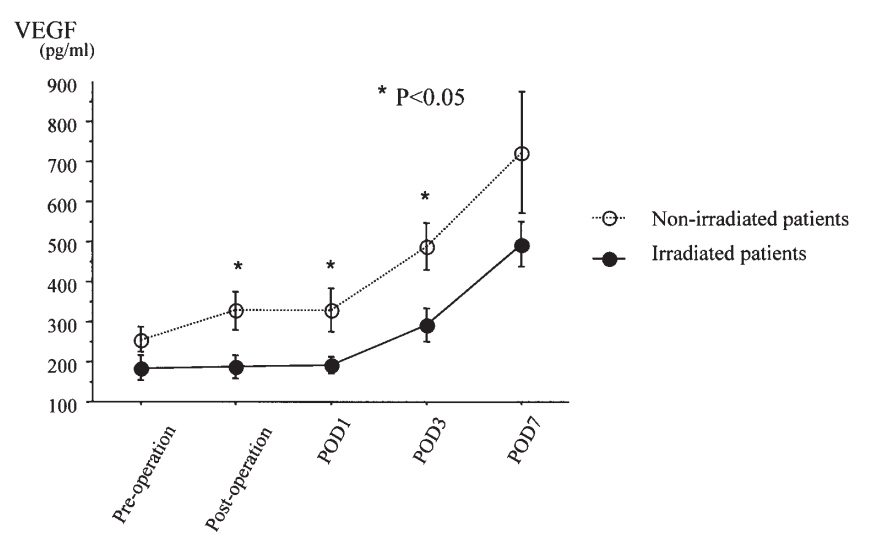

Figure 5. The post-operative circulating VEGF levels are significantly suppressed in irradiated patients compared with non-irradiated control patients.

Immunohistochemical staining of VEGF. Fig. 3 shows the immunohistochemical staining of VEGF in cancer tissue. VEGF was mainly localized in the cytoplasm or membrane of the cancer cell. Stromal cells were not stained.

VEGF levels in human tissue. Although the VEGF levels in human tissue were not significantly enhanced after chemo- radiation (Fig. 4a), the VEGF levels corrected for the number of viable cells, using the radiation effect (tissue VEGF concentration/radiation effect), were significantly increased $(\mathrm{p}<0.001)$ (Fig. 4b).

VEGF levels in serum. Fig. 5 shows the circulating levels of VEGF, before and after surgery, in patients with or without chemo-radiotherapy. The serum concentrations of VEGF increased gradually soon after surgery until POD 7 . In the control group, post-operative serum concentration of VEGF showed same alteration as the irradiated group. However, irradiated patients had significantly lower levels of circulating VEGF after surgery compared with the control group.

\section{Discussion}

Recently, with the success of a combined modality approach in the post-operative setting, there has been increased interest in preoperative chemo-radiotherapy for resectable rectal cancer. Neo-adjuvant chemo-radiotherapy is advantageous in its enhancement of locoregional control by eliminating microscopic residual disease around the primary tumor and in the draining lymphatics. There is also a possible impact on overall survival. By contrast, it may be disadvantageous due to its potent stimulation of angiogenesis, through molecules 
such as VEGF, leading to possible distant metastasis. Normally, VEGF is expressed weakly in a wide variety of human tissues; however, high expression levels can be detected at sites where angiogenesis is required, such as in fetal tissue and the vast majority of tumors (23). VEGF and its receptor are also expressed at high levels in metastatic human colon cancers and in tumor-associated endothelial cells (14). Takahashi et al (24) showed that the VEGF expression levels in patients with lymph node-negative colon cancer were significantly related to the time to recurrence, while Gascinu et al (15) noted that p53 and VEGF were both expressed in node-positive rectal cancer. The majority of disease-free patients showed p53-negative and VEGF-negative tumors, while distant metastases were seen in patients with VEGF positive tumors (15).

In the current study, the induction of VEGF expression after exposure to chemo-radiation was observed in vitro. Increased local levels of VEGF were also found in patients after preoperative chemo-radiotherapy. We focused on the induction of VEGF derived from residual cancer cells, since pathological effects of various degrees were seen in all patients and, immunohistochemically, VEGF was mainly localized in the cytoplasm or at the membrane of cancer cells. Consequently, the VEGF level per residual cancer cell was significantly increased in patients after chemoradiotherapy.

The prognostic impact of VEGF serum levels in patients with colon cancer has been evaluated in several studies (2528). Nakayama et al (25) reported on elevated circulating levels of VEGF in patients with advanced disease and in patients who experienced tumor recurrence. Although they showed that mean VEGF levels tend to decrease postoperatively compared with preoperative concentrations, Werther et al (26) evaluated post-operative circulating VEGF as a prognostic marker in colon cancer patients. Thus, both tumor-specific and circulating VEGF expression levels correlate with a poor prognosis. However, the relationship between local expression and circulating values remains to be fully investigated. Circulating VEGF levels reflect the total amount of VEGF produced systemically, whereas local VEGF expression is mainly influenced by the local status of cancer. Post-operative circulating VEGF levels are influenced by the cancer status and surgical stress, and may also be influenced by the effects of chemo-radiation. Thus, we evaluated the perioperative serum VEGF levels until postoperative day 7 , in both irradiated and non-irradiated control patients. Our results showed that serum concentrations of VEGF increased gradually soon after chemo-radiotherapy until POD 7. The serum concentration of VEGF in the control group also showed same alteration; however, irradiated patients had lower levels of VEGF after surgery than those in the control group. That is, systemic VEGF levels, which have a possible impact on distant metastasis, may be influenced not by chemo-radiation per se, but by its volume reduction effect.

In conclusion, preoperative chemo-radiotherapy enhanced tumor-specific VEGF expression, especially in individual cancer cells, both in vitro and in vivo. However, it did not necessarily enhance systemic VEGF expression, possibly because of tumor volume reduction induced by chemo-radio- therapy. The current results suggest that there may be no need to worry about a possible enhancement of distant metastasis through the VEGF pathway, by chemo-radiotherapy for rectal cancer, when a sufficient radiation effect is obtained; however, we need to further investigate whether VEGF expression after chemo-radiotherapy correlates with actual tumor recurrence.

\section{References}

1. NIH Consensus Conference: Adjuvant therapy for patients with colon and rectal cancer. JAMA 264: 1444-1450, 1990.

2. Watanabe T, Kiyomatsu T, Kanazawa T, et al: Chemoradiotherapy for rectal cancer: current status and perspectives. Int $\mathrm{J}$ Clin Oncol 9: 475-483, 2004.

3. Frykholm GJ, Glimelius B and Pahlman L: Preoperative or postoperative irradiation in adenocarcinoma of the rectum: final treatment results of a randomized trial and an evaluation of late secondary effects. Dis Colon Rectum 36: 564-572, 1993.

4. Pahlman L and Glimelius B: Pre- or post-operative radiotherapy in rectal and rectosigmoid carcinoma. Report from a randomized multicenter trial. Ann Surg 211: 187-195, 1990.

5. Hyams DM, Mamounas EP, Petrelli N, et al: A clinical trial to evaluate the worth of preoperative multimodality therapy in patients with operable carcinoma of the rectum: a progress report of National Surgical Breast and Bowel Project Protocol R-03. Dis Colon Rectum 40: 131-139, 1997.

6. Sauer R, Becker H, Hohenberger W, et al: German Rectal Cancer Study Group. Preoperative versus postoperative chemoradiotherapy for rectal cancer. N Engl J Med 351: 1731-1740, 2004.

7. Camma C, Giunta M, Fiorica F, Pagliaro L, Craxi A and Cottone M: Preoperative radiotherapy for resectable rectal cancer: a meta-analysis. JAMA 284: 1008-1015, 2000.

8. Colorectal Cancer Collaborative Group: Adjuvant radiotherapy for rectal cancer: a systematic overview of 8,507 patients from 22 randomised trials. Lancet 358: 1291-1304, 2001.

9. Trial SRC: Improved survival with preoperative radiotherapy in resectable rectal cancer. Swedish Rectal Cancer Trial. N Engl J Med 336: 980-987, 1997.

10. Ahmad NR and Nagle D: Long-term results of preoperative radiation therapy alone for stage $\mathrm{T} 3$ and $\mathrm{T} 4$ rectal cancer. $\mathrm{Br} \mathrm{J}$ Surg 84: 1445-1448, 1997.

11. Grann A, Feng C, Wong D, et al: Preoperative combined modality therapy for clinically resectable uT3 rectal adenocarcinoma. Int J Radiat Oncol Biol Phys 49: 987-995, 2001.

12. Pucciarelli S, Friso ML, Toppan P, et al: Preoperative combined radiotherapy and chemotherapy for middle and lower rectal cancer: preliminary results. Ann Surg Oncol 7: 38-44, 2000.

13. Yancopoulos GD, Davis S, Gale NW, Rudge JS, Wiegand SJ and Holash J: Vascular-specific growth factors and blood vessel formation. Nature 407: 242-248, 2000.

14. Takahashi Y, Kitadai Y, Bucana CD, Cleary KR and Ellis LM: Expression of vascular endothelial growth factor and its receptor, KDR, correlates with vascularity, metastasis and proliferation of human colon cancer. Cancer Res 55: 3964-3968, 1995.

15. Cascinu S, Graziano F, Catalano V, et al: An analysis of p53, BAX and vascular endothelial growth factor expression in nodepositive rectal cancer. Relationships with tumour recurrence and event-free survival of patients treated with adjuvant chemoradiation. Br J Cancer 86: 744-749, 2002.

16. Gorski DH, Beckett MA, Jaskowiak NT, et al: Blockage of the vascular endothelial growth factor stress response increases the antitumor effects of ionizing radiation. Cancer Res 59: 3374-3338, 1999.

17. Park JS, Qiao L, Su ZZ, et al: Ionizing radiation modulates vascular endothelial growth factor (VEGF) expression through multiple mitogen activated protein kinase dependent pathways. Oncogene 20: 3266-3280, 2001.

18. Nozue $\mathrm{M}$, Isaka $\mathrm{N}$ and Fukao $\mathrm{K}$ : Over-expression of vascular endothelial growth factor after preoperative radiation therapy for rectal cancer. Oncol Rep 8: 1247-1249, 2001.

19. Ojima E, Inoue $Y$, Watanabe $\mathrm{H}$, et al: The optimal schedule for 5 -fluorouracil radiosensitization in colon cancer cell lines. Oncol Rep 16: 1085-1091, 2006. 
20. Kusunoki M, Yanagi H, Kotera H, et al: Effects of pharmacokinetic modulating chemotherapy using oral UFT and continuous venous 5-FU infusion on the prognosis of irradiated rectal carcinomas with p53 overexpression. Int J Oncol 13: 653-657, 1998.

21. Kusunoki M, Yanagi H, Kamikonya N, et al: Significant effect of preoperative brachytherapy on survival rate after resection of rectal carcinoma. Int J Oncol 9: 645-651, 1996.

22. Japanese Society for Cancer of the Colon and Rectum: Japanese Classification of Colorectal Carcinoma. 1st edition. Kanehara and Co., Ltd., Tokyo, pp81-82, 1997.

23. Dvorak HF, Brown LF, Detmar M and Dvorak AM: Vascular permeability factor/vascular endothelial growth factor, microvascular hyperpermeability, and angiogenesis. Am J Pathol 146: 1029-1039, 1995.

24. Takahashi Y, Tucker SL, Kitadai Y, et al: Vessel counts and expression of vascular endothelial growth factor as prognostic factors in node-negative colon cancer. Arch Surg 132: 541-546, 1997.
25. Nakayama Y, Sako T, Shibao K, et al: Prognostic value of plasma vascular endothelial growth factor in patients with colorectal cancer. Anticancer Res 22: 2437-2442, 2002.

26. Werther K, Sorensen S, Christensen IJ and Nielsen HJ: Danish RANX05 Colorectal Cancer Study Group. Circulating vascular endothelial growth factor six months after primary surgery as a prognostic marker in patients with colorectal cancer. Acta Oncol 42: 837-845, 2003.

27. Werther K, Christensen IJ and Nielsen HJ: Danish RANX05 Colorectal Cancer Study Group. Prognostic impact of matched preoperative plasma and serum VEGF in patients with primary colorectal carcinoma. Br J Cancer 86: 417-423, 2002.

28. De Vita F, Orditura M, Lieto E, et al: Elevated perioperative serum vascular endothelial growth factor levels in patients with colon carcinoma. Cancer 100: 270-278, 2004. 